intervention phase with $75 \%$ of inpatients having DNACPR decisions and $46 \%$ escalation plans documented. Safety huddles helped improve ward culture and spread lessons learnt from debriefing of the last 3 events.

Conclusion Early results show there has been a reduction in total 2222 calls in the pilot ward compared to the other wards. We believe this is through improved decision making and empowering frontline staff. This could be scaled-up in other wards to have greater impact on patient care.

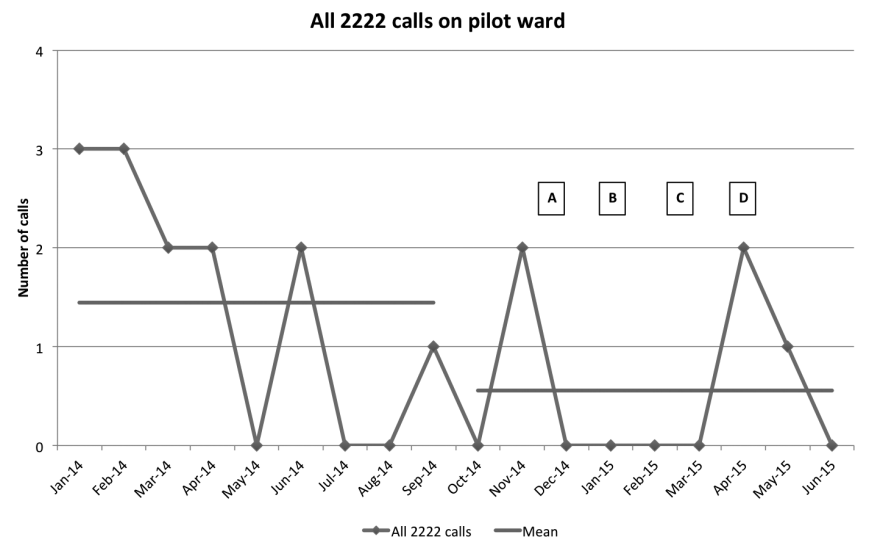

Abstract M7 Figure 1 Total number of 2222 calls on pilot ward. Interventions indicated by lettering (A): Staff survey, (B): Introduction of "deteriorating patient stamp", (C): Debriefing following any arrests, (D): Introduction of "Safety huddle". Mean of pre-intervention and intervention data, 1.44 and 0.56 respectively

\section{M8 PREVALENCE OF RESPIRATORY DISEASE IN SEVERE MORBID OBESITY}

SIW Lipworth, T Thevanathan, SW Copack, J Emmanuel. Barts and the London School of Medicine, London, UK

\subsection{6/thoraxjnl-2015-207770.435}

Introduction It is well established that asthma and obstructive sleep apnoea (OSA) are significantly more prevalent in obese vs. non-obese populations. To date however there is limited data on whether this risk is increased with severity of obesity as most studies classify all patients $>30 \mathrm{Kg} / \mathrm{m}^{2}$ simply as 'obese'. In addition, many existing studies' obese cohorts have fairly low BMI scores compared to patients attending specialist medical obesity services. Our study aims to examine the prevalence of these diseases in higher BMI groups, compare the relative risk of increasing obesity on prevalence of respiratory disease and investigate whether there is a synergistic effect of multiple demographic factors and severity of obesity.

Methods Data was collected from a total of 367 (of whom 159 had a BMI recorded) patient records attending a tier 4 obesity clinic over an 8 month period. Patients were divided into three groups according to severity of obesity, BMI 30-40, 40-50 and $>50 \mathrm{Kg} / \mathrm{m}^{2}$. Index of multiple deprivation (IMD) scores (mapped to postcodes) were used as a proxy of socioeconomic status.

Results $43 \%$ of our total cohort had OSA, including $75.7 \%$ of those with a BMI $>50$ (Multivariate logistic regression OR 10.4 (95\% CI $3.33-32.7, \mathrm{p}<0.001)$. In a chi-square analysis, this association was significant in both genders but stronger in males (Cramer's V 0.481 vs 0.305 ) and was significantly associated with a worse IMD score, being white and increasing age. 11.6\% of the cohort were asthmatic however there was no difference in prevalence between the groups OR 0.175 (95\% CI 0.019 $1.631, \mathrm{p}=0.126)$. There was however a significant co-effect of being male and increasing BMI in a multi-layer chi-square analysis $\mathrm{p}=0.044$.

Conclusions Our study highlights a very high prevalence of major respiratory diseases as co-morbidities in a severely obese population. Early data suggests a synergistic effect of Caucasian ethnicity, male gender and IMD score with increasing BMI on the risk of developing OSA (and Asthma for male gender). This is in contrast with our initial findings for Diabetes and Cardiovascular disease where the association is with Asian ethnicity.

\section{M9 LUNG HEALTH OF OPIATE USERS (LHOP): A PILOT STUDY TO ASSESS THE RESPIRATORY HEALTH OF OPIATE MISUSERS ATTENDING A COMMUNITY SUBSTANCE MISUSE CLINIC}

${ }^{1} \mathrm{~A}$ Pitt, ${ }^{2} \mathrm{C}$ Mitchell, ${ }^{2} \mathrm{~B}$ Colwell, ${ }^{3}$ I Appelqvist, ${ }^{3} \mathrm{~F}$ Ashby, ${ }^{4} \mathrm{C}$ Lloyd, ${ }^{4} \mathrm{~S}$ Gilbody, ${ }^{1} \mathrm{R}$ Lawson. ${ }^{1}$ Sheffield Teaching Hospitals NHS Foundation Trust, Sheffield, UK; ${ }^{2}$ Academic Unit of Primary Medical Care, The University of Sheffield, Sheffield, UK; ${ }^{3}$ South West Yorkshire NHS Trust/Phoenix Futures, Barnsley, UK; ${ }^{4}$ CLAHRC Mental Health and Co-Morbidities Stream, South Yorkshire, UK

\subsection{6/thoraxjnl-2015-207770.436}

Introduction MDTs promote harm reduction in the care of opiate misusers (OMUs) through the prescription of opiate substitute medication and by encouraging smoking rather than injecting (to reduce the risk of blood-bourne virus transmission and venous thromboembolism). The average life expectancy for a male OMU is 41 years and whilst evidence suggests that the current cohort of older OMUs are dying prematurely from nondrug related deaths, all-cause mortality studies rarely report the prevalence of chronic health problems. A recent case-controlled study reported a higher prevalence of asthma and COPD in OMUs after adjusting for tobacco consumption and other factors.

The study objective was to investigate the prevalence and illness burden of respiratory problems (asthma, COPD, symptomatic but undiagnosed lung disease) in patients with a history of current and/or past opiate misuse.

Methods Opportunistic clinic-based participant recruitment. Resting spirometry and researcher administered socio-demographic, inhaled drug use and validated respiratory patient reported outcome questionnaires: 1) prior diagnosis of asthma (ACT; mini-ARQoL) or COPD (CCQ); 2) respiratory health screening if no prior diagnosis (LFQ).

Results There were 36 participants (26 male; 10 female; aged 24-53). Only 8 had a diagnosis (all asthmatics); 35/36 smoked tobacco; 34/36 smoked heroin; 33/36 smoked cocaine; and 31/ 36 smoked cannabis. All asthmatics had poor control $(<13)$ on the ACT (median score 8 ) and frequent beta-agonist use (none used inhaled corticosteroids). Of the others, $22 / 28$ scored $\leq 18$ on LFQ suggesting high symptom burden and three of these had obstructive resting spirometry increasing the possibility of COPD.

Conclusions Chronic respiratory health in drug users is an under-researched area with few screening or high quality intervention studies evident. We identified a significant respiratory symptom burden within this OMU cohort. Most smoked tobacco, heroin, cocaine and cannabis. Asthmatics reported poor 
control and were potentially at risk of severe exacerbations, hospital admission and early progression to COPD.

Four further LHOp studies are planned: a pharmacy based intervention to improve asthma control/uptake of preventive interventions (smoking cessation; vaccinations); a case control study of GP asthma medication prescribing; prospective prognostic respiratory health cohort studies; and a qualitative study of asthmatic OMUs' perspectives on respiratory health and inhaler use.

\section{M10 THE DEVELOPMENT OF A VOCAL CORD DYSFUNCTION LARYNGOSCOPIC APPEARANCE SCALE}

${ }^{1} \mathrm{~J}$ Haines, ${ }^{1} \mathrm{~A}$ Vyas, ${ }^{1} \mathrm{C}$ Slinger, ${ }^{2} \mathrm{~L}$ Howell, ${ }^{3} \mathrm{SJ}$ Fowler. ${ }^{1}$ Lancashire Teaching Hospitals NHS Foundation Trust, Preston, UK; ${ }^{2}$ University of Central Lancashire, Preston, UK; ${ }^{3}$ Institute of Inflammation and Repair, University of Manchester, Manchester, UK

\subsection{6/thoraxjn-2015-207770.437}

Introduction Vocal cord dysfunction (VCD) typically involves abnormal vocal cord movement during inspiration. The recognised gold standard for diagnosis is fibreoptic laryngoscopy (FOL) during a symptomatic attack. Despite this there are no reported VCD FOL assessment scales to facilitate agreement in presentation, disease severity and treatment monitoring. Our VCD tertiary airways clinic receives over 300 referrals a year. We run a weekly diagnostic FOL list and identified the need for a VCD FOL classification for optimal care.

Aims To gain consensus for a VCD FOL appearance scale and identify its interrater reliability.

Methods An expert consensus group was convened comprising two respiratory consultant physicians and two respiratory speech and language therapists (SLTs). All have significant experience in VCD FOL interpretation. The group met, discussed and agreed on the VCD FOL appearance scale (Table 1). Two assessment teams were identified, each comprising a respiratory physician and a respiratory SLT. Each team rated patients, referred for FOL with a clinical suspicion of VCD, in three consecutive diagnostic FOL lists. All procedures were recorded and then blindly re-rated during playback by the other assessment team.

\begin{tabular}{ll}
$\begin{array}{l}\text { Abstract M10 Table } 1 \\
\text { appearance scale }\end{array}$ & Vocal cord dysfunction laryngoscopic \\
\hline Scale & Classification \\
\hline 0 & Normal vocal cord movements observed during respiration throughout assessment \\
1 & $\begin{array}{l}\text { Transient abnormal vocal cord movements observed during inspiration, with large } \\
\text { proportions of normal vocal cord movements during respiration }\end{array}$ \\
2 & $\begin{array}{l}\text { Mild abnormal vocal cord movements observed during respiration (up to } 50 \% \\
\text { vocal cord closure during inspiration) }\end{array}$ \\
3 & $\begin{array}{l}\text { Moderate abnormal vocal cord movements observed during inspiration (50\% or } \\
\text { more, but not total closure, i.e. gap still visible between the cords) }\end{array}$ \\
4 & Total apposition of the vocal cords (i.e. 100\% closure) observed during respiration \\
\hline
\end{tabular}

Results Eighteen patients received ratings; the mean (range) age was $51(19-80)$ and $78 \%$ were female. The assessing teams agreed on the rating for seven patients. For nine patients there was disagreement but adjacent classifications. Interrater agreement was performed using a weighted kappa $(1=$ complete agreement in classification; $0.5=$ disagreement but adjacent classifications; $0=$ disagreement and non-adjacent classifications). There was moderate agreement between the teams; 0.44 with a
$95 \%$ confidence interval of $0.18-0.70$. There was no bias between the assessment teams, as each had mean ratings for all patients of 2.4 .

Conclusions The VCD FOL appearance scale is a promising clinical assessment tool for the VCD population. We expected further interrater agreement; interestingly the majority of disagreement would not have changed management as classification still yielded a positive diagnosis. The differential maybe attributed to whether ratings were performed live or in playback, and this should be investigated. With further development, standardisation of application and robust validation it will be a useful assessment to direct appropriate management and facilitate accurate and consistent diagnosis.

\section{M11 A PRELIMINARY BIOPSYCHOSOCIAL MODEL OF VOCAL CORD DYSFUNCTION (VCD)}

${ }^{1} \mathrm{CC}$ Maskell, ${ }^{1} \mathrm{~N}$ Pargeter, ${ }^{1} \mathrm{~J}$ Fellows, ${ }^{1} \mathrm{~A}$ Mansur, ${ }^{2} \mathrm{R}$ Howard. ${ }^{1}$ Birmingham Heartlands Hospital, Birmingham, UK; ${ }^{2}$ University of Birmingham, Birmingham, UK

\subsection{6/thoraxjnl-2015-207770.438}

Introduction Vocal cord dysfunction (VCD) can have a significant impact on breathing ability and quality of life. ${ }^{1}$ Psychological factors can contribute to the onset and maintenance of the condition. ${ }^{2}$ Little has been written about the patient experience of VCD; therefore this study sought to understand what it is like to live with the condition and develop a preliminary model of VCD.

Methods Six adults diagnosed with VCD were interviewed and full transcripts of each interview were analysed via Interpretive Phenomenological Analysis. ${ }^{3}$ Six themes were generated reflecting the experiences of living with VCD. Next, the relationships between the themes were explored diagrammatically for each participant. Key phenomena or processes were fed into a summary cross-case diagram, which hypothesised how common aspects of the VCD experience may affect quality of life.

Results It is theorised that three key elements are interacting to influence quality of life: (1) bodily sensations, such as throat tightening, wheeze and cough can feed into a cycle of panic that exacerbates symptoms; (2) unpleasant or traumatic memories can lead to hyper-vigilance for symptoms, worry and social withdrawal; (3) enabling patients to understand, prepare and cope with the physical and mental aspects of VCD can reduce the impact of VCD on quality of life. Furthermore, environmental factors, such as the general lack of knowledge about the condition, can exacerbate negative experiences of VCD.

Conclusions We posit a biopsychosocial model of VCD which requires multidisciplinary treatment. In addition to speech and language therapy for throat-based symptoms, psychological interventions should be considered to address both thoughts about VCD and unpleasant memories. There is also an urgent need to improve and disseminate knowledge of the condition generally amongst medical professionals.

\section{REFERENCES}

1 Mansur AH. Vocal cord dysfunction. In: Palange P, Simonds AK, eds. European respiratory society handbook: Respiratory medicine. 2nd edn. Sheffield, England: European Respiratory Society, 2013: 274-279

2 Morris MJ, Oleszewski RT, Sterner JB, Allan PF. Vocal cord dysfunction related to combat deployment. Mil Med. 2013;178(11):1208-12

3 Willig C. Introducing qualitative research in psychology. Buckingham: Open University Press, 2009 